\title{
Ecologia de paisagens aplicada ao ordenamento territorial e gestão florestal: procedimento metodológicos
}

\author{
Luciano Cavalcante de Jesus FRANÇA ${ }^{1 *}$, Daniela Torres MORANDI ${ }^{2}$, Eduarda Soares MENEZES ${ }^{2}$, \\ Danielle Piuzana MUCIDA ${ }^{3}$, Marcelo Dutra da SILVA ${ }^{3}$, Gerson dos Santos LISBOA ${ }^{4}$
}

\author{
${ }^{1}$ Programa de Pós-Graduação em Engenharia Florestal, Universidade Federal de Lavras, Lavras, MG, Brasil. \\ ${ }^{2}$ Programa de Pós-Graduação em Ciência Florestal, Universidade Federal dos Vales do Jequitinhonha e Mucurí, \\ Diamantina, MG, Brasil. \\ ${ }^{3}$ Instituto de Oceanografia, Universidade Federal do Rio Grande, Rio Grande, RS, Brasil. \\ ${ }^{4}$ Centro de Formação em Tecno-ciências e Inovação, Universidade Federal do Sul da Bahia, Itabuna, BA, Brasil. \\ *E-mail: lucianodejesus@florestal.eng.br
}

Recebido em outubro/2018; Aceito em março/2019

RESUMO: No sentido de contribuir com o avanço das investigações científicas sobre Ecologia de Paisagens no contexto do ordenamento territorial e gestão florestal de recursos naturais, este estudo teve como objetivo reunir e discutir os principais conceitos e temáticas sobre a Ecologia de Paisagens, a abordar o uso da geotecnologia para análises estruturais e métricas da paisagem. A partir de revisão bibliográfica nacional e internacional, relativamente dos mais clássicos aos mais recentes estudos sobre o assunto. As bases dos periódicos científicos utilizados foram: Web of Science, Scielo (Nacional), Science Direct, Scopus, Google Scholar e Brazilian Digital Library of Theses. Foram destacados os conceitos fundamentais e aplicações nas áreas das ciências ambientais e florestais, no sentido de valorar estratégias e tomadas de decisões no planejamento e gestão ambiental rural. Neste trabalho, também foi apresentado o procedimento metodológico para cálculo das métricas da paisagem, por meio da extensão Patch Analyst, aplicada ao Software ArcGIS. Foram compiladas informações de 50 artigos científicos publicados no Brasil e exterior, onde constatou-se relevância significativa e viabilidade do uso dos índices e métricas da Ecologia de Paisagens para fins geográficos e ecológicos, embora, constatou-se que existem lacunas a serem preenchidas sobre o tema no Brasil, em relação às produções científicas, ainda escassas, sobretudo com análises mais concretas e direcionadas. O estudo permitiu a ampliação do conhecimento teórico sobre o tema no país e, espera-se que seja parte integrante e subsidiária para outros estudos sobre restauração ambiental, conexão de paisagens, mosaicos florestais e ordenamento territorial.

Palavras-chave: fragmentação florestal; métricas de paisagens; planejamento e gestão ambiental rural; patch analyst; sistema de informações geográficas.

\section{Landscape ecology applied to territorial order and forest management: methodological procedures}

\begin{abstract}
In order to contribute to the advancement of scientific research on Landscape Ecology in the context of spatial planning and forest management of natural resources, this study aimed to gather and discuss the main concepts and themes on Landscape Ecology, to address the use of geotechnology for structural analysis and landscape metrics. Based on national and international bibliographic review, relatively from the most classic to the most recent studies on the subject. The bases of the scientific journals used were: Web of Science, Scielo (National), Science Direct, Scopus, Google Scholar and Brazilian Digital Library of Theses. Key concepts and applications in the areas of environmental and forest sciences were highlighted in order to evaluate strategies and decision making in rural environmental planning and management. In this work, we also presented the methodological procedure for calculating the landscape metrics, through the Patch Analyst extension, applied to the ArcGIS Software. We compiled information from 50 scientific articles published in Brazil and abroad, where significant relevance and feasibility of the use of indexes and metrics of Ecology of Landscapes for geographic and ecological purposes was verified, although, it was verified that there are gaps to be filled on the theme in Brazil, in relation to the scientific productions, still scarce, especially with more concrete and directed analyzes. The study allowed the expansion of theoretical knowledge about the subject in the country and is expected to be an integral and subsidiary to other studies on environmental restoration, connection of landscapes, forest mosaics and land use planning.
\end{abstract}

Keywords: forest fragmentation; landscape metrics; rural environmental planning and management; patch analyst; geographic information system. 


\section{INTRODUÇÃO}

Ao longo da história, o conceito de paisagem evoluiu de forma significativa. No século XIX o naturalista Alexander Von Humboldt introduziu o termo paisagem na discussão científica da Geografia Física moderna e da Geobotânica (RITTER; MORO, 2012). Mais recentemente, vários estudiosos vêm a reforçar definições importantes na linha de investigação da Ecologia de Paisagens, sobretudo nas duas últimas décadas, tais como Metzger (2001); Metzger et al. (2007); Paudel; Yuan (2012); Cheung et al. (2016) e Ockendon et al. (2018).

A crescente preocupação com manejo dos recursos naturais tem gerado demandas por investigações científicas que atentem aos problemas relativos ao ordenamento territorial e gestão espacial destes recursos. Tornou-se, portanto, fundamental a caracterização da paisagem e, para isso são utilizados diversos métodos e técnicas de análises métricas da paisagem (ver tópico 1.2), de modo a tornar compreensível a dinâmica de sua estrutura, frente às mudanças impostas aos cenários, estudos de planejamento ambiental e à tomada de decisões, tais como projetos de licenciamento ambiental, zoneamentos ambientais, definições de malhas urbanas e rurais, definições de corredores ecológicas e unidades de conservação, dentre outras ações de intervenções que pode ser subsidiadas a partir do conhecimento da estrutura da paisagem. fragmentação de habitats é considerada a principal mudança ambiental impulsionadora das perdas de biodiversidade (LUSTIG et al., 2015). Neste sentido, conhecer a interação entre os elementos que compõem a estrutura da paisagem tem se mostrado uma das principais estratégias da agenda ambiental, em todo o mundo.

Embora a temática ainda se encontre escassa na literatura (DALLOZ et al., 2017) devido ao fato de estar sendo organizada e solidificada assim como pelo fato do tema ser recente no Brasil (PIVELLO; METZGER, 2007) a Ecologia das Paisagens apresenta duas vertentes de estudo, a geográfica e a ecológica (ver tópico 1.1). A maior parte dos estudos associam-se, principalmente, aos aspectos geográficos da ecologia de paisagens no que tange à ordenação territorial, pouco prestando-se a discutir processos ecológicos aplicados às múltiplas áreas da paisagem florestal.

Em estudo clássico, Metzger (2001) propõe uma definição integradora do conceito de paisagem, tratando-a como um mosaico heterogêneo formado por unidades interativas da paisagem. Para o autor, a heterogeneidade existe para, ao menos, um fator, segundo um observador em uma determinada escala de observação. A problemática em questão baseia-se na escassez de estudos científicos teóricos sobre a integração de análises de dinâmica da ecologia da paisagem aplicadas aos estudos de gestão de florestas e ordenamento territorial e ambiental.

Nesse sentido, levanta-se a seguinte pergunta: Qual a importância de considerar-se a ecologia de paisagens em projetos e planejamento ambiental? Sua resposta baseia-se na necessidade de elencar e analisar estudos já realizados e mais recentes sobre o tema, especialmente sobre métodos e procedimentos utilizados em análises de paisagens para diversos fins em estudos ecológicos. Ritter; Moro (2012) destacam a importância não apenas de descrever padrões e dinâmicas espaciais, mas a busca do entendimento de efeitos ecológicos em resposta às hipóteses científicas de forma quantitativa.
Essa revisão teve como objetivo principal reunir os principais conceitos e temáticas sobre Ecologia de Paisagens, através de revisão bibliográfica nacional e internacional, dos mais clássicos aos mais recente sobre o assunto, bem como elucidar os procedimentos metodológicos para cálculo das métricas de paisagens a partir da extensão em SIG Pacth Analyst.

Utilizou-se como base de pesquisa para essa revisão, as seguintes palavras chaves: paisagem, fragmentação florestal, métricas de paisagens, ordenamento territorial e ambiental, ecologia de paisagens, Patch Analyst, sistema de informações geográficas. As bases dos periódicos científicos utilizadas foram: Web of Science, Scielo (Nacional), Science Direct, Scopus, Google Scholar e Brazilian Digital Library of Theses. Assim, espera-se que esta revisão de literatura possa ser subsidiária à novos estudos de restauração ambiental, gestão florestal, dentre outras ações de ordenamento do território que utilizam-se das avaliações em ecologia de paisagens, bem como contribuir com a agregação de mais informações e conhecimentos literários acerca de paisagens. Quatro tópicos são abordados e discutidos neste estudo, sendo eles: (1.1) Enfoques da ecologia de Paisagens; (1.2) Métricas de paisagens podem ser eficazes no ordenamento territorial e ambiental? (1.3) As ferramentas para análise de paisagens em SIG: o uso do Patch Analyst e; (1.4) Procedimento metodológico para o Patch Analyst.

\subsection{Enfoques da Ecologia de Paisagens}

Foi a partir da década de 1980, que efetivamente, uma série de eventos impulsionou o desenvolvimento da Ecologia de Paisagens no mundo, com a criação em 1981, da Associação Internacional de Ecologia de Paisagens (IALE) e, em 1987, da revista Landscape Ecology, sob gestão da IALE e, a realização dos congressos internacionais quadrienais da IALE, que ocorrem desde o ano de 1981 (URBAN et al., 1997).

Há, basicamente, dois enfoques dentro da Ecologia de Paisagens (METZGER, 2001): (i) o geográfico, que privilegia o estudo da influência do homem sobre a paisagem e (ii) o ecológico, que enfatiza a importância do contexto espacial sobre os processos ecológicos e a importância destas relações em termos de conservação biológica. Ambos os enfoques muitas vezes mesclam-se em suas similaridades, apesar do primeiro alinhar-se à utilidade na gestão do território e o segundo valorizar a perspectiva do organismo.

A Ecologia de Paisagens surgiu na Europa, estritamente no contexto da Geografia Física e da ciência da vegetação. Adicionalmente, recebeu grande influência da Geografia Humana, focada no planejamento do uso da terra, linha de investigação de abordagem geográfica (KOBLITZ et al., 2011), tendo sido paralela e consequentemente, investigada cientificamente noutras regiões do mundo.

A abordagem norte-americana, baseada principalmente na ecologia de ecossistemas e na modelagem espacial, teve seu avanço beneficiado com o advento das imagens de satélite, vinculando-se ao Sensoriamento Remoto e Sistema de Informações Geográficas. Facilidades de tratamento de imagens e de análises geoestatísticas também propiciaram o desenvolvimento de estudos quantitativos do funcionamento ambiental, os quais consideram modelos estatísticos que levam em conta variáveis temporais e espaciais em diversas escalas (RITTER; MORO, 2012). Já a abordagem sul-americana, tem se baseado principalmente no arcabouço conceitual do 
brasileiro Mezger (2001), com a definição integradora de paisagem, já bastante difundida.

A Ecologia da Paisagem envolve o estudo de padrões de paisagem, suas mudanças e interações entre manchas limitadas a um mosaico ao longo do tempo por meio de quantificação (MCGARIGAL; MARKS, 1995). Baseia-se, em grande parte, na ideia de que a padronização da paisagem em elementos (manchas), influenciam fortemente características ecológicas, incluindo flora e fauna.

Neste sentido, estudos na área de Ecologia de Paisagem têm assumido amplo espaço no meio científico mundial nos últimos anos, por meio do uso de áreas, índices, densidades e percentagens descritores de análise da estrutura, função e mudança da paisagem, denominados de métricas de paisagem (MCGARIGAL; MARKS, 1995, MANDER; MARJA, 2013).

1.2. Métricas de paisagens podem ser eficazes no ordenamento territorial e ambiental?

Segundo Nowosad; Stepinski (2018) houve um avanço significativo em investigações de padrões de paisagens em nível global. Muitas destas investigações, aplicam métricas de paisagens para: entendimento da integridade espacial, delimitação de corredores e conectividade da paisagem (SALOMÃO et al., 2018; SCHACKELFORD et al., 2018; SIMPKINS et al., 2018); análise do planejamento espacial e proteção dos serviços prestados pela natureza (INKOOM et al., 2018); subsidiar gestão de unidades de conservação (SILVA; LIMA, 2016; MIZAEL, 2016), estratégias para recuperação de áreas degradadas (PAULA et al, 2015; TOLEDO et al., 2018), ambientes de mineração (LEI et al., 2016) e paisagens ameaçadas pelo avanço da exploração de petróleo e gás no sistema costeiros (MARTINS, 2017).

Além disso, as métricas de paisagem aplicam-se no manejo de fauna silvestre, como por exemplo em estudo de Kosicki (2018) que analisou, a partir da configuração da paisagem, a densidade de espécies de aves especialistas e generalistas. Neste mesmo sentido, Morelli et al. (2018) avaliaram associações de métricas de paisagem com a diversidade de aves e métricas de comunidades, concluindo que a diversidade taxonômica relacionou-se à métrica da diversidade, explicada pelas métricas de paisagem. Índices da paisagem podem, ainda, auxiliar no planejamento de espaços verdes em ambientes urbanos (ZHANG; WANG, 2006; LIU; YANG, 2015; LIU et al., 2017) e outras diversas formas de cobertura da terra (PARENTE; VOLIN, 2016).

Os gestores florestais precisam identificar novas maneiras de prever com rapidez e precisão a composição da paisagem florestal pós-perturbação (SAVAGE et al., 2017). Nesse contexto, para o manejo florestal, as métricas têm sido utilizadas principalmente no monitoramento de mudanças e eficiência de planos de manejo (SANO et al., 2009). Isso ocorre uma vez que distintas métricas são capazes de caracterizar diferentes aspectos de padrões estruturais de uma floresta (KILGORE et al., 2013). Plexida et al. (2014) utilizaram métricas para analisar a heterogeneidade espacial de diferentes paisagens em uma abordagem multiescala em regiões mediterrâneas. Já Santos et al. (2018) as utilizaram para apresentar propostas de criação de corredores ecológicos para uma região de Mata Atlântica.

Pirovani et al. (2015) e Salomão et al. (2018) analisaram e quantificaram, por meio do uso de índices e métricas de ecologia de paisagem, mudanças estruturais ocorridas em período de cerca de 36-37 anos em unidades de conservação e entornos nos estados do Espírito Santo e Minas Gerais, respectivamente. Seus resultados apontaram para um aumento na fragmentação da paisagem nos entornos das unidades de conservação. Prado et al (2017) a partir de informações sobre área, perímetro, índice de circularidade, área de borda e isolamento, avaliaram a condição de fragilidade ambiental de fragmentos florestais em um parque estadual na Amazônia.

A Geotecnologia aliada a Ecologia de Paisagens é ainda capaz de selecionar potenciais fragmentos florestais para coleta de sementes tendo por finalidade ações de restauração ecológica (SANTOS et al., 2016). Análises do estado atual de cobertura florestal são estudos efetivos no contexto do uso das métricas de paisagens, como observado por Silva el al. (2015) em área de Mata Atlântica em Minas Gerais, cujo estudo foi eficaz para avaliar o estado atual da conservação da paisagem. Saito et al. (2016), em estudo sobre análise temporal da cobertura florestal, concluíram que as métricas de paisagens associadas à mineração de dados são eficazes para aumentar aplicabilidades de dados de Sensoriamento Remoto, sendo importante estratégia para tomadas de decisões.

Teixeira et al. (2016) destacam que os conceitos e aplicações de Ecologia de Paisagens podem ser aplicados em grandes áreas geográficas, corroborando trabalho de Silva et al. (2015a). Ambos ressaltam que a Ecologia da Paisagem é relevante para a definição de estratégias de ocupação e uso conservacionista em unidades territoriais como bacias hidrográficas. Miller; Brewer (2018) em estudo realizado no Reino Unido, utilizaram as métricas de paisagens para estimar índices de inundação em bacias hidrográficas urbanizadas.

No contexto do manejo e gestão de bacias hidrográficas é possível, ainda, avaliar-se por meio das métricas, a correlação dinâmica da composição da paisagem e a fragmentação dos habitats com a qualidade das águas superficiais, conforme estudo realizado por Xie et al. (2018), na China.

Métricas de paisagens também podem ser poderosas ferramentas em projetos de Estudo de Impacto Ambiental (EIA). A incorporação de conceitos da Ecologia de Paisagens em diagnósticos e relatórios podem qualificar e auxiliar processo de tomada de decisão sobre a viabilidade ambiental e ecológica de grandes empreendimentos (KOBLITZ et al. 2011). Xu et al. (2015) utilizaram-se da Ecologia de Paisagens para modelar a dinâmica do sistema de crescimento industrial e a fragmentação da paisagem na China, mostrando-se eficiente, ao apresentar taxa de erro de modelo inferior a 5\%, explicando então coerentemente as relações entre desenvolvimento industrial e as paisagens chinesas.

No Brasil, evidenciou-se uma lacuna relativa a investigações científicas no campo da Ecologia de Paisagens. Esta deficiência é constatada e corroborada no estudo desenvolvido por $\mathrm{Wu}$ (2012), em levantamento sobre os últimos 30 anos de estudos na temática, onde quantificaram-se os 20 artigos científicos mais citados do mundo entre 1987 e 2011, sendo todos eles desenvolvidos por pesquisadores estrangeiros.

Em pesquisa avançada realizada na base Web of Science, com uso das palavras chave: Landscape Ecology and Landscape Metrics, para o período entre os anos 2000 e 2019, foram encontrados 1.834 registros de artigos desenvolvidos pelo mundo, que de forma direta ou indireta utilizaram-se dos termos ou métodos de ecologia de paisagens. Para o Brasil, os registros na mesma base de indexação, indicam 130 artigos 
publicados no mesmo período, com as palavras chave: Landscape Ecology and Landscape Metrics and Brazil.

1.3. As ferramentas para análise de paisagens em SIG: o uso do Patch Analyst

Existem inúmeras possibilidades de ferramentas de Sistema de Informações Geográficas (SIG) que auxiliam na análise de paisagens, sobretudo, na avaliação de métricas. Muitos estudos apresentam avaliações semelhantes com uso da extensão Patch Analyst para o ArcGIS (PAUDEL; YUAN, 2012). Outros estudos utilizaram o Software FRAGSTATS (LIU et al. 2016; NIESTEROWICZ; STEPINSKI, 2016; PENG et al., 2016), pioneiro em estudos de paisagens (MCGARIGAL; MARKS, 1995). Apesar disso, apresentam limitações quanto aos resultados para áreas fragmentadas, uma vez que podem criar bordas artificiais em determinados fragmentos (HASSETT et al., 2012).

Outra extensão é o V-LATE, muito difundido e utilizado em alguns trabalhos e elaborada para análises em ArcGIS (SANTOS et al., 2016; ADAMCZYK; TIEDE, 2017). Apesar disso, o FRAGSTATS e Patch Analyst são, atualmente, os principais softwares para cálculo de métricas de paisagens. Adamczyk; Tiede (2017) propõem um novo comando para cálculo de métricas implementado na caixa de ferramentas Python, no ArcGIS 10.1, por apresentar interface gráfica de fácil utilização e possibilidade de cálculos de lotes. Para outros softwares, tais como o QGIS, há uma ferramenta capaz de calcular, de forma automatizada, uma variedade de métricas básicas e avançadas de paisagens, o plugin LecoS (JUNG, 2016).

Santos et al. (2016) citam várias métricas na descrição de padrões espaciais a partir de resultados obtidos pelo uso de softwares de Geoprocessamento e Sensoriamento Remoto. Os autores, em estudo em bacia hidrográfica no Espírito Santo, verificaram que a combinação de técnicas como classificação de imagens, ecologia da paisagem florestal e análise multicritério (MCA) permitiu avaliar a evolução temporal e espacial da fragmentação florestal assim como os índices métricos e os potenciais fragmentos florestais para a colheita de determinadas sementes, contribuindo para a proteção florestal e manejo da Mata Atlântica. Baral et al. (2014) utilizaram-se da extensão Patch Analyst para avaliação espacial e mapeamento da biodiversidade em paisagem fragmentada na Austrália.

Em região de Mata Atlântica, no Espírito Santo, Silva et al. (2015b) obtiveram resultados eficazes por meio de métricas pela ferramenta Patch Analyst, concluindo qualidade ambiental dos remanescentes florestais encontra-se altamente comprometida, devido à elevada fragmentação.

\subsection{Procedimento metodológico para o Patch Analyst}

É necessário para todo o procedimento, a instalação da extensão Patch Analyst, para ArcGIS (Download: http://www.cnfer.on.ca/SEP/patchanalyst/Patch5_2_Install.ht $\underline{m})$. O procedimento metodológico para cálculo das métricas de paisagens no ArcMap, utilizando a extensão gratuita Patch Analyst (REMPEL et al., 2012), é apresentado a seguir e em fluxograma da Figura 1.

Partindo-se de posse de imagens de satélites classificadas quanto ao uso e ocupação da terra, por parte do operador, executa-se, inicialmente, a conversão dos dados raster de entrada para formato vetorial shapefile (To polygon). A partir da conversão, as etapas para cálculo das métricas compreendem:

( $1^{\circ}$ Passo) Inicialização da Estatística Espacial: Patch Analyst $>$ Spatial Statistics

( $2^{\circ}$ Passo) Inserção do Layer e definição da análise desejada: Adiciona-se o layer do mapa em questão > Analyze By > Define-se Landscape ou Class, uma vez que é possível analisar por classes individuais ou em nível de paisagem; selecionar Gridcode tendo em vista que as classes encontram-se neste formato;

( $3^{\circ}$ Passo) Output Table Name: salva-se o nome do arquivo de saída, que será a tabela de atributos gerada;

(4 Passo) Advanced Options: Analyze Vectors As $>$ Raster ou Vector, comumente seleciona-se Vector, neste caso por se estar utilizando um vetor poligonal;

( $5^{\text {a }}$ Passo) Pressiona-se o comando Run, onde é gerada a análise estatística espacial das métricas da paisagem. Automaticamente será gerada uma tabela com as siglas referentes às métricas de tamanho, borda e forma.

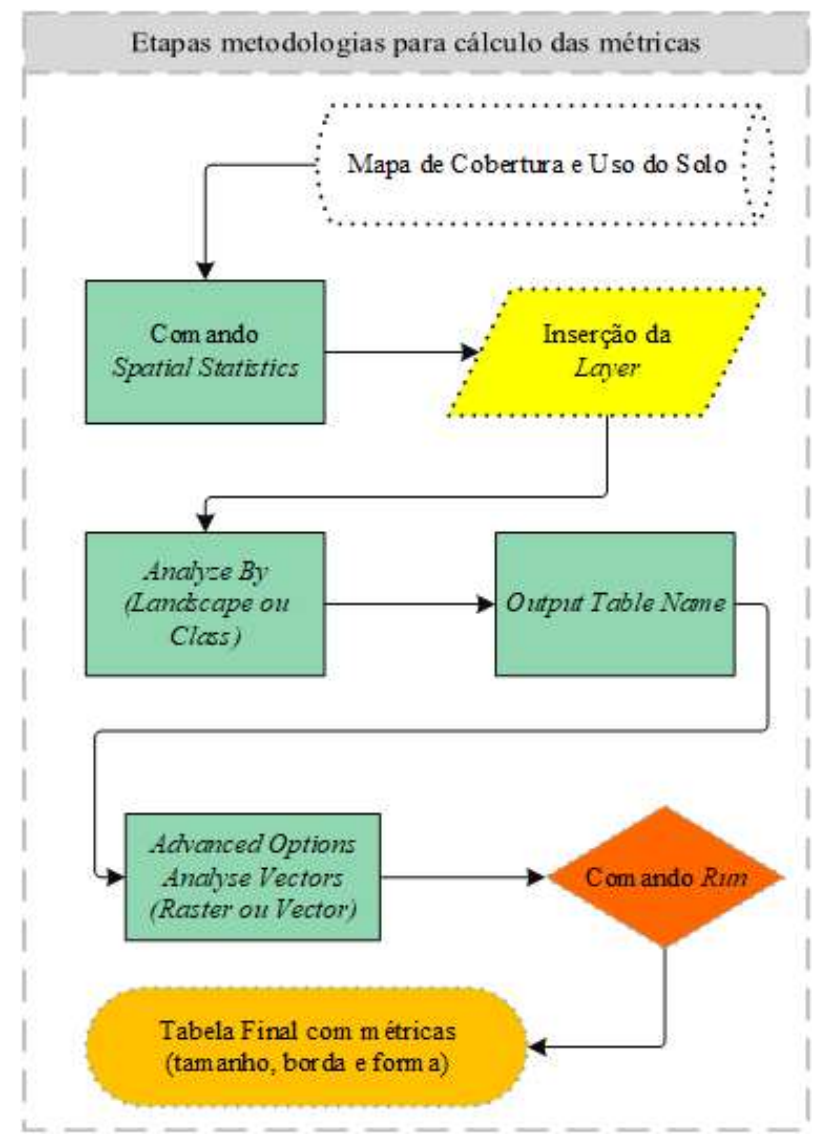

Figura 1. Fluxograma dos procedimentos metodológicos para calcular métricas de paisagens pelo Patch Analyst.

Figure 1. Flowchart with methodological procedures for the calculation of landscape metrics by Patch Analyst.

Os índices calculados pelo Patch Analyst, para uma determinada paisagem, são caracterizados pelos grupos e descrições apresentadas na Tabela 1 . 
Tabela 1. Métricas de Ecologia da Paisagem geradas no Patch Analyst, adaptado de McGarigal; Marks (1995).

Table 1. Landscape Ecology Metrics generated in Patch Analyst, adapted from McGarigal; Marks (1995)

\begin{tabular}{|c|c|c|c|c|}
\hline Grupo & Sigla & Métrica & Unidade & Descrição \\
\hline Área & $\mathrm{CA}$ & Classe area & Hectare (ha) & $\begin{array}{l}\text { Área total da classe; representa a soma de todas as manchas de determinada } \\
\text { classe de uso da terra. }\end{array}$ \\
\hline \multirow{10}{*}{ Tamanho } & TLA & Landscape area & Hectare (ha) & $\begin{array}{l}\text { Área total da paisagem; expressa a soma de todas as manchas de todas as } \\
\text { classes. Expressa a área total em estudo. }\end{array}$ \\
\hline & NumP & $\begin{array}{l}\text { Number of } \\
\text { patches }\end{array}$ & Adimensional & $\begin{array}{l}\text { Número total de manchas; expressa o número total de manchas por classe de } \\
\text { uso da terra. }\end{array}$ \\
\hline & MPS & Mean patch size & Hectare (ha) & $\begin{array}{l}\text { Tamanho médio das manchas; expressa o tamanho médio das manchas por } \\
\text { classe de uso da terra. }\end{array}$ \\
\hline & MedPS & $\begin{array}{l}\text { Median patch } \\
\text { size }\end{array}$ & Hectare (ha) & $\begin{array}{l}\text { Tamanho mediano das manchas; expressa o tamanho mediano das manchas } \\
\text { por classe de uso da terra. }\end{array}$ \\
\hline & PSSD & $\begin{array}{l}\text { Patch size } \\
\text { standard } \\
\text { deviation }\end{array}$ & Hectare (ha) & $\begin{array}{l}\text { Desvio padrão do tamanho das manchas; expressa a variação do tamanho } \\
\text { das manchas em torno do valor médio para cada classe de uso da terra. }\end{array}$ \\
\hline & PSCoV & $\begin{array}{l}\text { Patch size } \\
\text { coefficient of } \\
\text { variance }\end{array}$ & $\begin{array}{l}\text { Porcentagem } \\
\qquad(\%)\end{array}$ & $\begin{array}{l}\text { Coeficiente de variação do tamanho da mancha; expressa o desvio padrão } \\
\text { em porcentagem, ou seja, da variação para cada classe de uso da terra. }\end{array}$ \\
\hline & TE & Total edge & Metro $(\mathrm{m})$ & Comprimento total do perímetro (borda)ppara cada classe de uso da terra. \\
\hline & ED & Edge density & $\mathrm{m} / \mathrm{ha}$ & $\begin{array}{l}\text { Densidade de borda; expressa a relação entre perímetro (te) de cada classe } \\
\text { pela área total da paisagem (tla) }\end{array}$ \\
\hline & MPE & $\begin{array}{l}\text { Mean patch } \\
\text { edge }\end{array}$ & $\mathrm{m}$ & $\begin{array}{l}\text { Média do perímetro (borda); expressa o comprimento médio do perímetro } \\
\text { (te) das manchas para cada tipo de classe. É calculado pela divisão do valor } \\
\text { total (te) pelo número total de manchas (nump). }\end{array}$ \\
\hline & MPAR & $\begin{array}{l}\text { Mean } \\
\text { perimeter-area } \\
\text { ratio }\end{array}$ & $\mathrm{m} / \mathrm{m}^{2}$ & $\begin{array}{l}\text { Média da relação perímetro/área. Assemelha-se ao edge density (ed). É } \\
\text { calculada a relação entre o perímetro (te) de cada classe pela área total classe } \\
\text { (ca) dividido pelo número de manchas (nump). }\end{array}$ \\
\hline \multirow{4}{*}{ Forma } & MSI & $\begin{array}{l}\text { Mean shape } \\
\text { index }\end{array}$ & Adimensional & $\begin{array}{l}\text { Indicador médio de forma; expressa o quanto a mancha é próxima de um } \\
\text { círculo. A métrica efetua a soma do perímetro de todas as manchas e a divide } \\
\text { pelo quadrado da área da classe de uso. Expressa que quanto mais próximo } \\
\text { de } 1 \text { for o valor, mais próxima ao formato circular é a mancha analisada. }\end{array}$ \\
\hline & AWMSI & $\begin{array}{l}\text { Area weighted } \\
\text { mean shape } \\
\quad \text { index }\end{array}$ & Adimensional & $\begin{array}{l}\text { Indicador médio de forma ponderada pela área; expressa o quanto a mancha } \\
\text { é próxima de um círculo. A métrica efetua a soma do perímetro de todas as } \\
\text { manchas dividido pelo quadrado da área da classe de uso. O resultado é } \\
\text { dividido pelo número de manchas da classe. Quanto mais próximo de } 1 \text { for } \\
\text { o valor, mais próxima ao formato circular é a mancha analisada. }\end{array}$ \\
\hline & MPFD & $\begin{array}{l}\text { Mean patch } \\
\text { fractal } \\
\text { dimension }\end{array}$ & Adimensional & $\begin{array}{l}\text { Dimensão fractal média da mancha. Trata-se de uma métrica que expressa a } \\
\text { complexidade de forma da mancha. Valores próximos a } 1 \text { representam } \\
\text { perímetros simples e valores próximos a } 2 \text { indicam perímetros complexos, } \\
\text { baseados na forma. }\end{array}$ \\
\hline & AWMPFD & $\begin{array}{l}\text { Area weighted } \\
\text { mean patch } \\
\text { fractal } \\
\text { dimension }\end{array}$ & Adimensional & $\begin{array}{l}\text { Dimensão fractal média da mancha ponderado pela área; expressa a } \\
\text { complexidade de forma da mancha, sendo que valores próximos de } 1 \\
\text { representam perímetros simples e valores próximos de } 2 \text { indicam perímetros } \\
\text { complexos, baseados na forma. Grandes manchas possuem tendência a } \\
\text { maior complexidade que pequenas manchas. Por isso, a métrica analisa a } \\
\text { complexidade independentemente do tamanho das manchas. Normalmente } \\
\text { o resultado será menor do que no mpfd. }\end{array}$ \\
\hline Proximidade & $\mathrm{MNN}$ & $\begin{array}{l}\text { Average } \\
\text { distance from } \\
\text { nearest } \\
\text { neighbor }\end{array}$ & Metro (m) & $\begin{array}{l}\text { A distância média do vizinho mais próximo é a média dessas distâncias para } \\
\text { classes individuais ao nível de classe e a distância média da classe vizinha } \\
\text { mais próxima ao nível de paisagem. }\end{array}$ \\
\hline \multirow{6}{*}{ Área Central } & TCA & $\begin{array}{l}\text { Total central } \\
\text { area }\end{array}$ & Hectare (ha) & O tamanho total das manchas. \\
\hline & $\mathrm{MCA}$ & $\begin{array}{l}\text { Middle Central } \\
\text { area }\end{array}$ & Hectare (ha) & O tamanho médio das manchas de área central \\
\hline & $\mathrm{NCA}$ & $\begin{array}{l}\text { Number of } \\
\text { central areas }\end{array}$ & Adimensional & $\begin{array}{l}\text { Número total de áreas centrais dentro da paisagem ou dentro de cada mancha } \\
\text { ao nível de classe. }\end{array}$ \\
\hline & CASD & $\begin{array}{l}\text { Standard } \\
\text { deviation of the } \\
\text { central area }\end{array}$ & Hectare (ha) & Raiz da variância da média das áreas centrais \\
\hline & $\mathrm{CACoV}$ & $\begin{array}{l}\text { Coefficient of } \\
\text { variation of } \\
\text { center area }\end{array}$ & $\begin{array}{l}\text { Porcentagem } \\
\qquad(\%)\end{array}$ & $\begin{array}{l}\text { Representa a variabilidade em tamanho das áreas centrais disjuntas em } \\
\text { relação à área central média. }\end{array}$ \\
\hline & TCAI & $\begin{array}{l}\text { Total central } \\
\text { area index }\end{array}$ & $\begin{array}{l}\text { Porcentagem } \\
\qquad(\%)\end{array}$ & Medida da quantidade relativa de área central na paisagem. \\
\hline
\end{tabular}


Estudos de Ecologia da Paisagem aplicados em etapas de planejamento, diagnóstico e manutenção de projetos ambientais em diferentes condições, podem auxiliar significativamente a gestão florestal de recursos naturais e ao ordenamento territorial, como apresentado em estudos de caso. Todavia, evidenciou-se para o Brasil uma lacuna em produção científica avançada no assunto, apresentando apenas alguns especialistas clássicos na linha de pesquisa, assim como o emprego da expressão 'ecologia de paisagens' ainda se apresenta pouco utilizado no que tange às ciências ambiental e florestal no país.

Quanto ao procedimento metodológico para cálculo das métricas pelo Patch Analyst em ambiente do software ArcGIS, verificou-se que a manipulação da base de dados e etapas metodológicas apresentam-se relativamente fáceis e flexíveis para aplicação, sendo importante a correta interpretação dos resultados, para uma eficaz análise espacial.

\section{CONSIDERAÇÕES FINAIS}

A partir dessa revisão, pôde-se constatar a grande relevância e viabilidade do uso de métricas vinculadas ao entendimento da Ecologia de Paisagens, seja do ponto de vista geográfico ou ecológico, seja em estudos regionais ou locais. Os estudos apresentados para diferentes aplicações em várias ecorregiões mundiais, destacam a amplitude do tema nas ciências ambientais e florestais.

\section{AGRADECIMENTOS}

$O$ presente trabalho foi realizado com apoio da Coordenação de Aperfeiçoamento de Pessoal de Nível Superior - Brasil (CAPES) - Código de Financiamento 001.

\section{REFERÊNCIAS}

ADAMCZYK, J.; TIEDE, D. ZonalMetrics - a Python toolbox for zonal landscape structure analysis. Computers \& Geosciences, Laramie, v. 99, p. 91-99, 2017. DOI: https://dx.doi.org/10.1016/j.cageo.2016.11.005

BARAL, H.; KEENAN, R. J.; SHARMA, S. K.; STORK, N. E.; KASEL, S. Spatial assessment and mapping of biodiversity and conservation priorities in a heavily modified and fragmented production landscape in northcentral Victoria, Australia. Ecological Indicators, Coimbra, v. 36, p. 552-562, 2014. DOI: https://dx.doi.org/10.1016/j.ecolind.2013.09.022

CHEUNG, A. K. L.; BRIERLEY, G.; O'SUlLIVAN, D. Landscape structure and dynamics on the Qinghai-Tibetan Plateau. Ecological Modelling, Towson, v. 339, p. 7-22, 2016. https://dx.doi.org/10.1016/j.ecolmodel.2016.07.015

DALLOZ, M. F.; CROUZEILLES, R.; ALMEIDA-GOMES, M.; PAPI, B.; PREVEDELLO, J. A. Incorating landscape ecology metrics into environmental impact assessment in the Brazilian Atlantic Forest. Perspectives in ecology and conservation, São Paulo, v. 15, n. 3, p. 216-220, 2017. DOI: https://dx.doi.org/10.1016/j.pecon.2017.07.002

HASSETT, E. M.; STEHMAN, S. V.; WICKHAM, J. D. Estimating landscape pattern metrics from a sample of land cover. Landscape Ecology, Prague, v. 27, n. 1, p. 133-149, 2012. DOI: https://dx.doi.org/10.1007/s10980-011-9657-4
INKOOM, J. N.; FRANK, S.; GREVE, K.; WALZ, U.; FURST, C. Suitability of different landscape metrics for the assessments of patchy landscapes in West Africa. Ecological Indicators, Coimbra, v. 85, p. 117-127, 2018. DOI: https://dx.doi.org/10.1016/j.ecolind.2017.10.031

JUNG, M. LecoS - A python plugin for automated landscape ecology analysis. Ecological Informatics, Adelaide, v. 31, p. 18-21, 2016.2 DOI: https://dx.doi.org/10.1016/j.ecoinf.2015.11.006

KILGORE, M. A.; SNYDER, S. A.; BLOCK-TORGERSON, K.; TAFF, S. J. Challenges in characterizing a parceled forest landscape: why metric, scale, threshold, and definitions matter. Landscape and Urban Planning, Michigan, v. 110 , p. 36-47, 2013. DOI: https://dx.doi.org/10.1016/j.landurbplan.2012.09.009

KOBLITZ, R.; PEREIRA JUNIOR, S. J.; AJUZ, R. C. de Ecologia de Paisagens e Licenciamento Ambiental. Natureza \& Conservação, Goiânia, v. 9, n. 2, p. 244-248, 2011. DOI: https://dx.doi.org/10.4322/natcon.2011.033

KOSICKI, J. Z. Are Landscape Configuration Metrics Worth Including When Predicting Specialist and Generalist Bird Species Density? A Case of the Generalized Additive Model Approach. Environ Model Assess, Brisbane, v. 23, n. $2, \quad$ p. 193-202, 2018. DOI: https://dx.doi.org/10.1007/s10666-017-9575-1

LEI, K.; PAN, H.; LIN, C. A landscape approach towards ecological restoration and sustainable development of mining areas. Ecological Engineering, Prague, v. 90, p. 320-325, 2016.

DOI: https://dx.doi.org/10.1016/j.ecoleng.2016.01.080

LIU, M.; HU, Y. M.; CHUN, L. L. Landscape metrics for three-dimensional urban building pattern recognition. Applied Geography, Georgia, v. 87, p. 66-72, 2017. DOI: https://dx.doi.org/10.1016/j.apgeog.2017.07.011

LIU, T.; YANG, X. Monitoring land changes in an urban area using satellite imagery, GIS and landscape metrics. Applied Geography, Georgia, v.56, p.42-54, 2015. DOI: https://dx.doi.org/10.1016/j.apgeog.2014.10.002

LIU, Y.; WEI, X.; LI, P.; LI, Q. Sensitivity of correlation structure of class- and landscape- level metrics in three diverse regions. Ecological Indicators, Coimbra, v. 64, p.9-19, 2016.

DOI: https://dx.doi.org/10.1016/j.ecolind.2015.12.021

LUSTIG, A. STOUFFER, D. B.; ROIGÉ, M.; WORNER, S. P. Towards more predictable and consistent landscape metrics across spatial scales. Ecological Indicators, Coimbra, v. 57, p. 11-21. DOI: https://dx.doi.org/10.1016/j.ecolind.2015.03.042

MANDER, E.U.U.; MARJA, R. Trends in the use of landscape spatial metrics as landscape indicator: A review. Ecological Indicators, Coimbra, v. 28, p. 100-106, 2013. DOI: https://dx.doi.org/10.1016/j.ecolind.2012.07.018

MCGARIGAL, K.; MARKS, B. J. FRAGSTATS: spatial pattern analysis program for quantifying landscape structure. Gen. Tech. Rep. PNW-GTR-351. Portland, OR: US Department of Agriculture, Forest Service, Pacific Northwest Research Station, 1995. v. 351. 122 p. Disponível em: $<$ https://www.fs.fed.us/pnw/pubs/gtr_351.pdf $>$. Acesso em: 15 de junho 2018. 
METZGER, J.O.; FONSECA, M. A. da.; OLIVEIRA FILHO, F. J. B. de; MARTENSEN, A. C. O uso de modelos em ecologia de paisagens. Megadiversidade, Belo Horizonte, v. 3, n.1-2, p. 64-73, 2007. Acesso em: 14 de setembro 2018.

METZGER, J. P. O que é ecologia de paisagens? Biota Neotropica, Campinas, v. 1, n. 1, p. 1-9, 2001. DOI: http://dx.doi.org/10.1590/S1676-06032001000100006

MILLER, J.D.; BREWER, T. Refining flood estimation in urbanized catchments using landscape metrics. Landscape and Urban Planning, Michigan, v. 175, p. 34-49, 2018. DOI:

https://dx.doi.org/10.1016/j.landurbplan.2018.02.003

MORELLI, F.; BENEDETTI, Y.; SÍMOVÁ, P. Landscape metrics as indicators of avian diversity and community measures. Ecological Indicators, Coimbra, v. 90, p. 132141 , 2018.

DOI: https://dx.doi.org/10.1016/j.ecolind.2018.03.011

NIESTEROWICZ, J.; STEPINSKI, T. F. On using landscape metrics for landscape similarity search. Ecological Indicators, Coimbra, v. 64, p. 20-30, 2016. DOI: https://dx.doi.org/10.1016/j.ecolind.2015.12.027

NOWOSAD, J.; STEPINSKI, T. F. Global inventory of landscape patterns and latent variables of landscape spatial configuration. Ecological Indicators, Coimbra, v. 89, p. 159-167, 2018.

DOI: https://dx.doi.org/10.1016/j.ecolind.2018.02.007

OCKENDON, N; THOMAS, D. H. L.; CORTINA, J.; ADAMS, W. M.; AYKROYD, T.; BAROV, B.; BOITANI, L.; BONA, A.; BRANQUINHO, C.; BROMBACHER, M.; BURELL, C.; CARVER, S.; CRICK, H. Q. P.; DUGUY, B.; EVERETT, S.; FOKKENS, B.; FULLER, R. J.; GIBBONS, D. W.; SUTHERLAND, W. J. One hundred priority questions for landscape restoration in Europe. Biological Conservation, Montpellier, v. 221, p. 198-208, 2018. DOI: https://dx.doi.org/10.1016/j.biocon.2018.03.002

PARENTE, J. R.; VOLIN, J. C. Validating Landsat-based landscape metrics with fine-grained land cover data. Ecological Indicators, Coimbra, v. 60, p. 668-677, 2016. DOI: https://dx.doi.org/10.1016/j.ecolind.2015.08.009

PAUDEL, S.; YUAN, F. Assessing landscapes changes and dynamics using patch analysis and GIS modeling. International Journal of Applied Earth Observation and Geoinformation, Enschede, v. 16, p. 66-76, 2012. DOI: https://dx.doi.org/10.1016/j.jag.2011.12.003

PAULA, M.D.; GROENEVELD, J.; HUTH, A. Tropical forest degradation and recovery in fragmented landscapes - Simulating changes in tree community, forest hydrology and carbon balance. Global Ecology and Conservation, Yunnan, v. 3, p. 664-677, 2015. DOI: https://dx.doi.org/10.1016/j.gecco.2015.03.004

PENG, Y.; FENGTING QING, K. M.; XUE, D. Identification of the main factors determining landscape metrics in semiarid agro-pastoral ecotone. Journal of Arid Environments, Trelew, v. 124, p. 249-256, 2016. DOI: https://dx.doi.org/10.1016/j.jaridenv.2015.08.009

PIROVANI, D.B.; DA SILVA, A. G.; DOS SANTOS, A. R. Análise da paisagem e mudanças no uso da terra no entorno da RPPN Cafundó, ES. Cerne, v. 21, n. 1, p. $27-$ 35,2015 .
PIVELLO, V. R.; METZGER, J. P. Analysis of the research on Landscape Ecology in Brazil (2000-2005). Biota Neotrópica, Campinas, v. 7, n. 3, 2007. DOI: http://dx.doi.org/10.1590/S1676-06032007000300002

PLEXIDA, S. G.; SFOUGARUS, A. I.; ISPIKOUDIS, I. P..; PAPANASTASIS, V. P. Selecting landscape metrics as indicators of spatial heterogeneity - A comparison among Greek landscapes. International Journal of Applied Earth Observation and Geoinformation, Enschede, v. 26, p. 26-35, 2014. https://dx.doi.org/10.1016/j.jag.2013.05.001

PRADO, L. K. B.; SANTOS, A. F. A.; WOJCIECHOWSKI, J. C.; CLAUDINO, W. V.; EISENLOHR, M. A. C. Fragilidade ambiental dos fragmentos florestais do Parque Estadual Cristalino, por métricas da paisagem. Nativa, Sinop, v. 5, p. 548-554, 2017. DOI: http://dx.doi.org/10.5935/2318-7670.v05nespa13

RITTER, L. M. O.; MORO, R. S. Epistemological bases of landscape ecology. Journal of Biotechnology and Biodiversity, Gurupi, v. 3, n.3, p. 58-61, 2012.

REMPEL, R. S.; KAUKINEN, D.; CARR, A. P. Patch Analyst and Patch Grid. Ontario Ministry of Natural Resources. Centre for Northern Forest Ecosystem Research, Thunder Bay, Ontario, 2012.

SAITO, N. S.; ARGUELLO, F. V. P.; MOREIRA, M. A.; SANTOS, A. R. dos. EUGENIO, F. C.; FIGUEIREDO, A. C. Uso da geotecnologia para análise temporal da cobertura florestal. Cerne, Lavras, v. 22, n. 1, p. 11-18, 2016

DOI: http://dx.doi.org/10.1590/01047760201622011935

SALOMÃO, N.V. MACHADO, E. L. M.; PEREIRA, R. S.; FERNANDES, G. W.; GONZAGA, A. P. D.; MUCIDA, D. P.; SILVA, L. S. da. Structural analysis of a fragmented area in Minas Gerais State, Brazil. Anais da Academia Brasileira de Ciências, v. 90, n. 4, p.3353-3361, 2018. DOI: http://dx.doi.org/10.1590/0001-3765201820160828

SANO, M.; MYAMOTO, A.; FURUYA, N.; KOGI, K. Using landscape metrics and topographic analysis to examine forest management in a mixed forest, Hokkaido, Japan: Guidelines for management interventions and evaluation of cover changes. Forest Ecology and Management, Amsterdam, v. 257, p. 1208-1218, 2009. DOI: https://dx.doi.org/10.1016/j.foreco.2008.10.005

SANTOS, A.R.; RIBEIRO, C. A. A. S.; PELUZIO, T. M. de O.; PELUZIO, J. B. E.; QUEIROZ, V. T. de.; BRANCO, E. R. F.; LORENZON, A. S.; DOMINGUES, G. F.; MARCATTI, G. E.; CASTRO, N. L. M. de.; TEIXEIRA, T. R.; SANTOS, M. G. A. D. A. dos; MOTA, P. H. S.; SILVA, S. F. da.; VARGAS, R.; CARVALHO, J. R.; MACEDO, L. L.; ARAÚJO, C. da S.; ALMEIDA, S. L. H. de. Geotechnology and landscape ecology applied to the selection of potential forest fragments for seed harvesting. Journal of Environmental Management, Sint-KatelijneWaver, v. 183, p. 1050-1063, 2016. DOI: https://dx.doi.org/10.1016/j.jenvman.2016.09.073.

SANTOS, J.S. LEITE, C. C. C.; VIANA, J. C. C.; SANTOS, A. R. dos.; FERNANDES, M. M.; ABREU, V. de S.; NASCIMENTO, T. P. do.; SANTOS, L. S. dos.; FERNANDES, M. R. de M.; SILVA, G. F. da.; MENDONÇA, A. R. de. Delimitation of ecological corridors in the Brazilian Atlantic Forest. Ecological Indicators, Coimbra, v. 88, p. 414-424, 2018. DOI: https://dx.doi.org/10.1016/j.ecolind.2018.01.011. 
SAVAGE, S. L.; LAWRENCE, R. L.; SQUIRES, J. R. Mapping post-disturbance forest landscape composition with Landsat satellite imagery. Forest Ecology and Management, Amsterdam, v. 399, p. 9-23, 2017. DOI: https://dx.doi.org/10.1016/j.foreco.2017.05.017

SCHACKELFORD, N.; STANDISH, R. J.; LINDO, Z.; STARZOMSKI, B. M. The role of landscape connectivity in resistance, resilience, and recovery of multi-trophic microarthropod communities. Ecology, Califórnia, v. 99, n. $5, \quad$ p. 1164-1172, 2018. DOI: https://dx.doi.org/10.1002/ecy.2196

SILVA, K.G.; SANTOS, A. R. dos.; SILVA, A. G. da.; PELUZIO, J. B. E.; FIEDLER, N. C.; ZANETTI, S. S. Análise da dinâmica espaço temporal dos fragmentos florestais da sub-bacia hidrográfica do rio Alegre, ES. Cerne, Lavras, v. 21, n. 2, p. 311-318, 2015. DOI: https://dx.doi.org/10.1590/01047760201521021562

SILVA, R. A.; PEREIRA, J. A. A.; BARROS, D. M.; BORGES, L. A. C.; TEIXEIRA, M. D.; ACERBI-JR., F. W. Avaliação da cobertura florestal na paisagem de mata atlântica no ano de 2010, na região de Ouro Preto - MG. Cerne, Lavras, v. 21, n. 2, p. 301-309, 2015. DOI: https://dx.doi.org/10.1590/01047760201521021539

SIMPKINS, C. E.; DENNIS, T. E.; ETHERINGTON, T. R.; PERRY, G. L. W. Assessing the performance of common landscape connectivity metrics using a virtual ecologist approach. Ecological Modelling, Towson, v. 367, p.1323 , 2018.

DOI: https://dx.doi.org/10.1016/j.ecolmodel.2017.11.001

TEIXEIRA, F. Z.; COELHO, I. P.; LAUXEN, M. The need to improve and integrate science and environmental licensing to mitigate wild life mortality on roads in Brazil. Tropical Conservation Science, Thousand Oaks, v. 9, n. 1, p. 3442, 2016.

DOI:
TOLEDO, R. M.; SANTOS, R. F.; VERHEYEN, K.; PERRING, M. P. Ecological restoration efforts in tropical rural landscapes: Challenges and policy implications in a highly degraded region. Land Use Policy, Adelaide, v. 75, p. 486-493, 2018. DOI: https://dx.doi.org/10.1016/j.landusepol.2018.03.053

URBAN, D. L.; O'NEILL, R. V.; SHUGART, H. H. Landscape Ecology: a hierarchical perspective can help scientists understand spatial patterns. BioSicence, New Mexico, v. 37, p. 119-127, 1997.

XIE, Y.; YU, X.; NG, N. C.; LI, K.; FANG, L. Exploring the dynamic correlation of landscape composition and habitat fragmentation with surface water quality in the Shenzhen river and deep bay cross-border watershed, China. Ecological Indicators, Coimbra, v. 90, p. 231-246, 2018. DOI: https://doi.org/10.1016/j.ecolind.2017.11.051

XU, J.; KANG, J.; SHAO, I.; ZHAO, T. System dynamic modelling of industrial growth and landscape ecology in China. Journal of Environmental Management, SintKatelijne-Waver, v. 161, p. 92-106, 2015. DOI: https://dx.doi.org/10.1016/j.jenvman.2015.06.026

WU, J. key concepts and research topics in landscape ecology revisited: 30 years after the Allerton Park workshop. Landscape Ecology, Tempe, v. 28, n. 1, p. 1-11, 2012. DOI: https://dx.doi.org/10.1007/s10980-012-9836-y

ZHANG, L.; WANG, H. Planning an ecological network of Xiamen Island (China) using landscape metrics and network analysis. Landscape and Urban Planning, Michigan, v. 78, n. 4, p. 449-456, 2006. DOI: https://dx.doi.org/10.1016/j.landurbplan.2005.12.004 